\title{
Burden of comorbidities and medication use in childbearing women with rheumatic diseases: a nationwide population-based study
}

Min Kyung Chung ${ }^{1, *}$, Chan Hee Lee ${ }^{2, *}$, Jin Su Park ${ }^{2}$, Hyunsun Lim ${ }^{3}$, and Jisoo Lee ${ }^{1}$

\section{Burden of comorbidities and medication use in childbearing women with rheumatic diseases: a nationwide population-based study}

\begin{tabular}{ll|l|l|} 
Korean women with rheumatic diseases (RDs) \\
during their childbearing years
\end{tabular}

Received : February 15, 2021

Revised : April 1, 2021

Accepted: April 12, 2021
Correspondence to Jisoo Lee, M.D.

Division of Rheumatology, Department of Internal Medicine, Ewha Womans University College of Medicine, 1071 Anyangcheon-ro, Yangcheon-gu, Seoul 07985, Korea

Tel: +82-2-2650-6164, Fax: +82-2-2650-5272, E-mail: leejisoo@ewha.ac.kr

https://orcid.org/0000-0001-6279-7025

*These authors contributed equally to this work. 
Background/Aims: We aimed to estimate the prevalence of comorbidities and medication use in Korean women with rheumatic diseases (RDs) during their childbearing years.

Methods: We included women aged 20 to 44 years with seropositive rheumatoid arthritis (RA), systemic lupus erythematosus (SLE), and ankylosing spondylitis (AS) ( $n=41,547)$ and age-matched women without seropositive RA, SLE, and AS ( $n=$ 208,941) from the National Health Insurance Service-National Health Information Database (2009 to 2016). The prevalence of hypertension (HTN), hyperlipidemia (HLD), diabetes mellitus (DM), and cancer and the use of nonsteroidal anti-inflammatory drugs (NSAIDs), corticosteroids (CSs), and disease-modifying anti-rheumatic drugs (DMARDs) were estimated.

Results: Women of childbearing age with RDs were more likely to have at least one of the measured comorbidities than the controls (odds ratio [OR], 3.0; 95\% confidence interval [Cl], 2.9 to 3.1). The OR (95\% Cl) was 2.9 (2.8 to 3.0) for HTN, 2.8 (2.7 to 2.9$)$ for HLD, 1.4 (1.4 to 1.5) for DM, and 1.3 (1.3 to 1.4) for cancer. The SLE group had the highest prevalence and odds of all four measured comorbidities. Almost all (97.9\%) women of childbearing age with RDs were taking RD-related medications (NSAIDs, 81.6\%; CSs, 77.8\%; DMARDs, 87.3\%). The RD group was 13.8 times more likely to take NSAIDs and 68.2 times more likely to take CSs than the controls. Use of NSAIDs was more prevalent in RA and AS than SLE, whereas use of CSs and DMARDs was more prevalent in RA and SLE than AS.

Conclusions: Korean women with RDs have a greater burden of comorbidities and medication use during their childbearing years than women without RDs of the same age.

Keywords: Rheumatic diseases; Reproductive age; Comorbidity; Drug utilization

\section{INTRODUCTION}

Rheumatic diseases (RDs) such as rheumatoid arthritis (RA), systemic lupus erythematosus (SLE), and ankylosing spondylitis (AS) can affect women during their reproductive years [1-3]. As RDs are chronic inflammatory diseases requiring life-long management, disease- and treatment-related complications and various comorbidities associated with these diseases can significantly affect childbearing in women with RDs. The influence of disease activity on pregnancy outcomes in patients with RDs has been well documented. High disease activity has been shown to be associated with adverse pregnancy outcomes in patients with SLE, RA, and AS [4-6]. Moreover, medications used for disease control can affect fertility and pregnancy outcomes. Treatment with cyclophosphamide can result in permanent ovarian failure [7]. Exposure to methotrexate (MTX) and mycophenolate mofetil (MMF) have been consistently reported to increase the rate of miscarriage. However, whether there is a correlation between miscarriages and drugs, such as nonsteroidal anti-inflammatory drugs (NSAIDs), corticosteroids (CSs), cyclophosphamide, cyclosporine, and tacrolimus remain unclear because these drugs are used in combination therapy; thus, conclusions cannot be drawn regarding single-drug exposure [8]. In addition, various comorbidities can affect childbearing in patients with RDs. Cardiovascular disease (CVD) and cancer are known to be more prevalent in patients with RA, AS, and SLE than in the general population [9-11]. However, the effects of comorbidities associated with RDs on childbearing or pregnancy outcomes have not been studied. From the studies showing increased rates of obstetric and fetal complications in women with CVD [12], and decreased fertility and permanent ovarian failure in premenopausal women being treated for cancer [13], we can infer that women in childbearing age with RDs are at risk for infertility and adverse pregnancy outcomes.

Although the burden of the disease on women of childbearing age has been studied previously [14-16], few studies have evaluated the burden of comorbidities and treatment medications used by women with RDs during their childbearing years. Determining the nationwide prevalence of comorbidities and medication use in a specific age group of women of childbearing age with RDs may provide valuable information for understanding the socioeconomic burden imposed on this specific population group. Thus, we aimed to estimate the prevalence of comorbidities and medication use among Korean women with RDs in their childbearing years using a nationwide population database. In this study, we focused on two important comorbidities, namely CVD and cancer. To assess the CVD risk, we assessed the prev- 
alence of hypertension (HTN), diabetes mellitus (DM), and hyperlipidemia (HLD), which are traditional risk factors for CVD.

\section{METHODS}

\section{Study design and source population}

We conducted a retrospective cohort study using data from the Korean National Health Insurance Service-National Health Information Database (NHIS-NHID) from 2009 to 2016. The Korean NHIS is a single insurer that provides Coverage for almost the entire Korean population; $97 \%$ of the Korean population is enrolled in the NHIS program [17]. As of December 2014, the NHIS database included all inpatient and outpatient claims data and information of approximately 50 million Korean people [18]. The NHIS-NHID has four databases with collected data on participants' insurance eligibility, medical treatment, medical care institution, and general health examinations [18]. This study included women of childbearing age (defined as women between the ages of 20 and 44 years) whose data had been collected during the period from January 1, 2009, to December 31, 2016. This study was approved by the Institutional Review Board of the National Health Insurance Service Ilsan Hospital (Institutional Review Board number: NHIMC 2020-06-011) and conducted according to the principles of the Declaration of Helsinki. As the database used in this study contains anonymized data for research purposes, informed consent was not required.

\section{Study population}

The study population included a cohort of cases and controls. Cases included in the RD group were women from the NHIS-NHID with seropositive RA, SLE, and AS between the ages of 20 and 44 years identified with the diagnostic codes of M05, M32, and M45 based on the International Classification of Diseases (ICD), 10th revision code, during the study period. RA, SLE, and AS were selected as they are representative RDs that occur frequently in women of reproductive age. In 2009, the government of the Republic of Korea subsidized medical expenses for patients with rare and intractable diseases through a copayment assistance policy called the Individual Copayment Beneficiaries Program (ICBP), and seropositive RA, SLE, and AS were designated as the rare diseases covered by this program. Under this ICBP system, the NHIS established a registration program that includes codes for the target disease classified per the Korean Standard Classification of Diseases (KCD)-7 (based on the ICD-10), date of definitive diagnosis, and tests performed for the confirmation of the diagnosis. We used data from January 1, 2009, with the assumption that all patients with seropositive RA, SLE, and AS have been accurately coded since ICBP registration requires fulfillment of classification criteria for definitive diagnosis.

Control subjects were age-matched women without diagnostic codes for seropositive RA (M05), SLE (M32), or AS (M45) designated in the NHIS-NHID during the study period; they were randomly sampled in a 1:5 ratio. Control subjects were given an index date that was the same as the date of diagnosis of the matched cases.

\section{Outcome measures}

Four specific comorbidities chosen for prevalence estimation were HTN, HLD, DM, and cancer. Comorbidities were identified using ICD-10 codes (I10-12/15 for HTN, E78 for HLD, E10-14 for DM, and C* for cancer). As cancer was one of the four major conditions eligible for insurance coverage benefits with a registration requirement to receive the benefit, it was assumed to be accurately coded [19]. In the RD group, only comorbidities occurring after the date of definitive diagnosis of RD were included. Likewise, comorbidities occurring after the index date were included in the control subjects. Frequently prescribed medications for RDs, including NSAIDs, CSs, and disease-modifying anti-rheumatic drugs (DMARDs), were chosen to estimate their use. DMARDs included in the analysis were azathioprine, cyclosporine, hydroxychloroquine, sulfasalazine, tacrolimus, MTX, leflunomide, MMF, mizoribine, adalimumab, etanercept, golimumab, infliximab, ustekinumab, rituximab, abatacept, and tocilizumab. Prescribed medications were identified based on the generic names of the drugs from the medical treatment database, and medications consecutively prescribed for over 90 days were assessed. Topical CSs were excluded from the analysis.

\section{Statistical analysis}

A descriptive analysis was performed to summarize the baseline characteristics of the participants. Categorical variables are presented as frequencies and percentages, and continuous variables are presented as mean with standard deviation (SD). To compare the prevalence of comorbidities 
between the RD and control groups, the chi-square test was used, and odds ratios (ORs) with 95\% confidence intervals (Cls) were adjusted for age, income level, and residential district. A $p<0.05$ was considered statistically significant. All statistical analyses were performed using SAS version 9.4 (SAS Institute, Cary, NC, USA).

\section{RESULTS}

\section{Characteristics of the study population}

A total of 41,547 women with RDs and 208,941 controls, both of childbearing age were identified. The RD group included 23,756 (57.2\%) women with RA; 12,756 (30.47\%) women with SLE; and 5,035 (12.1\%) women with AS. The demographic characteristics of subjects with RDs and controls are shown in Table 1. The mean \pm SD age was $35.0 \pm$ 6.5 years in both the RD and control groups. More women in the RD group than in the control group were enrolled in medical aid, had lower income level, and had their residence in a special city district.

\section{Prevalence of comorbidities}

Table 2 shows the prevalence of comorbidities, i.e., HTN, HLD, DM, and cancer, among women with RDs and control subjects of childbearing age. The prevalence of all four measured comorbidities was significantly higher in the RD group than in the control group ( $p<0.0001$ for all). Women of childbearing age with RDs were more likely to have at least one of the measured comorbidities (OR, 3.0; 95\% Cl, 2.9 to 3.1). Likewise, the RD group was more likely to have HTN (OR, 2.9; 95\% Cl, 2.8 to 3.0), HLD (OR, 2.8; 95\% Cl, 2.7 to 2.9), DM (OR, 1.4; $95 \% \mathrm{Cl}, 1.4$ to 1.5), and cancer (OR, 1.3; $95 \% \mathrm{Cl}, 1.3$ to 1.4$)$ than the age-matched controls.

When the prevalence of comorbidities was compared between the women with different RDs, significant differences were found among the RD groups. SLE had the highest prevalence of all four comorbidities followed by RA and AS (Fig. 1). ORs for having at least one of the measured comorbidities were $5.4(95 \% \mathrm{Cl}, 5.2$ to 5.7$)$ for SLE, $2.3(95 \%$

Table 1. Baseline characteristics of study population: RDs and controls

\begin{tabular}{|c|c|c|c|}
\hline Characteristic & $\operatorname{RDs}(n=41,547)$ & Controls $(n=208,941)$ & $p$ value \\
\hline Age, yr & $35.0 \pm 6.5$ & $35.0 \pm 6.5$ & - \\
\hline $20-24$ & $3,387(8.2)$ & $17,073(8.17)$ & \\
\hline $25-29$ & $6,050(14.6)$ & $30,466(14.6)$ & \\
\hline $30-34$ & $7,877(19.0)$ & $39,649(19.0)$ & \\
\hline $35-39$ & $11,457(27.6)$ & $57,579(27.6)$ & \\
\hline $40-44$ & $12,776(30.8)$ & $64,174(30.7)$ & \\
\hline Income level $^{\mathrm{a}}$ & & & $<0.001$ \\
\hline$<3$ & $12,869(31.0)$ & $69,408(34.4)$ & \\
\hline $3-7$ & $16,853(40.6)$ & $87,833(43.5)$ & \\
\hline$>7$ & $9,092(21.9)$ & $44,724(22.1)$ & \\
\hline Insurance & & & $<0.001$ \\
\hline Employee & $25,791(62.9)$ & $129,338(61.9)$ & \\
\hline Self-employment & $13,635(33.2)$ & $75,563(36.2)$ & \\
\hline Medical aid & $1,602(3.9)$ & $4,040(1.9)$ & \\
\hline Residential district & & & $<0.001$ \\
\hline City, province & $8,951(21.5)$ & $46,642(22.3)$ & \\
\hline Metropolitan city & $10,490(25.3)$ & $53,465(25.6)$ & \\
\hline Special city & $6,725(52.7)$ & $108,834(52.1)$ & \\
\hline
\end{tabular}

Values are presented as mean \pm SD or number (\%).

$\mathrm{RD}$, rheumatic disease.

a Income levels are presented by decile method (group $>7$ refers to low-income group). 


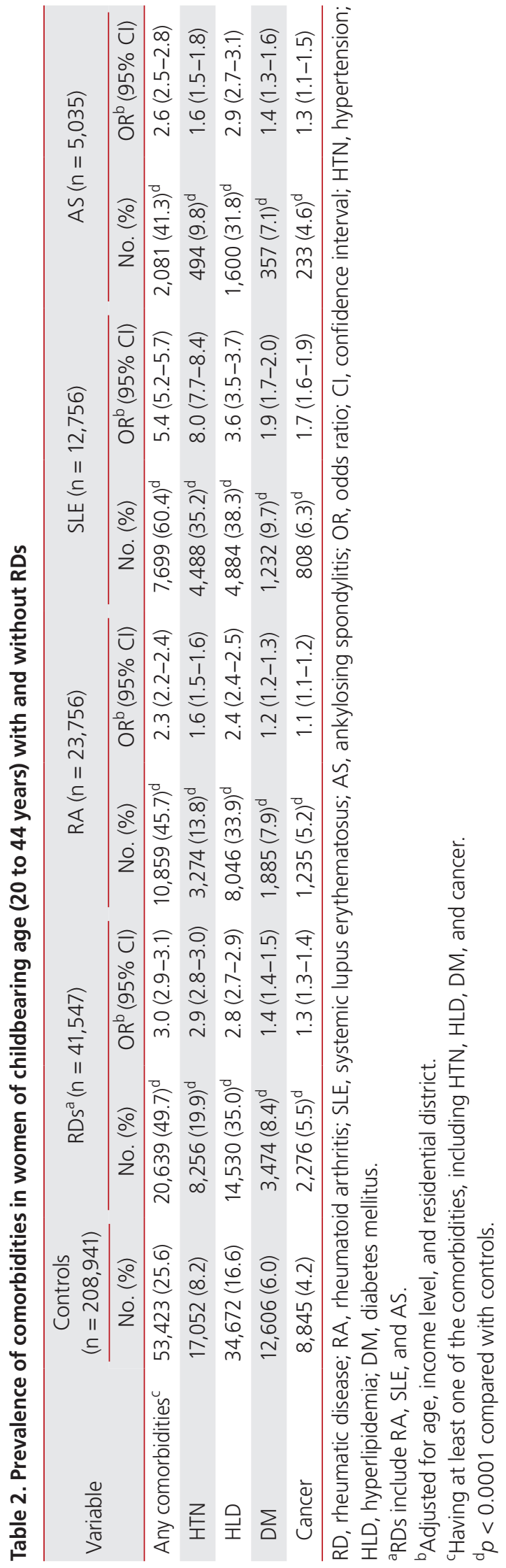

$\mathrm{Cl}, 2.2$ to 2.4 ) for $\mathrm{RA}$, and 2.6 (95\% Cl, 2.5 to 2.8 ) for AS. Women with SLE had the highest odds of having HTN (OR, 8.0; $95 \% \mathrm{Cl}, 7.7$ to 8.4), HLD (OR, 3.6; $95 \% \mathrm{Cl}, 3.5$ to 3.7 ), $\mathrm{DM}(\mathrm{OR}, 1.9 ; 95 \% \mathrm{Cl}, 1.7$ to 2.0$)$, and cancer (OR, 1.7; $95 \%$ $\mathrm{Cl}, 1.6$ to 1.9 ) (Table 2).

\section{Medication use}

Almost all (97.9\%) women of childbearing age with RDs were taking RD-related medications. NSAIDs were prescribed in $81.6 \%$ of patients, CSs in $77.8 \%$, and DMARDs

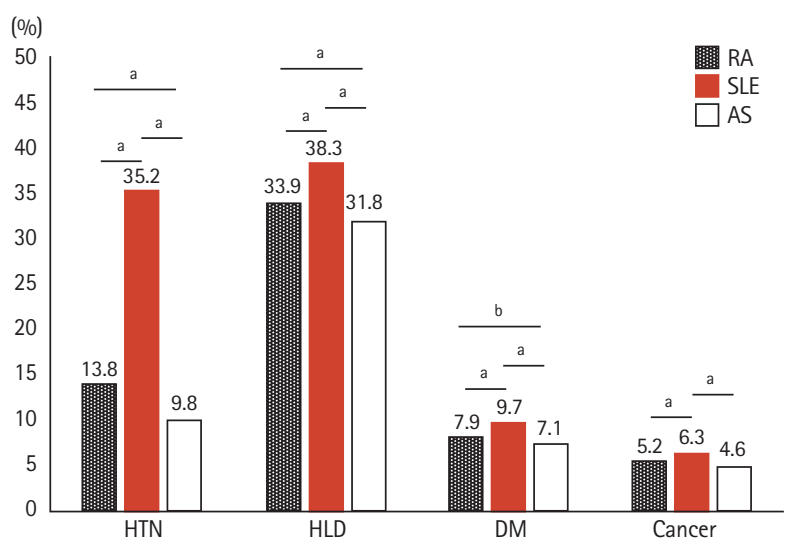

Figure 1. Comparison of comorbidity rates among women of childbearing age (20 to 44 years) with different rheumatic diseases. HTN, hypertension; HLD, hyperlipidemia; DM, diabetes mellitus; RA, rheumatoid arthritis; SLE, systemic lupus erythematosus; AS, ankylosing spondylitis. ${ }^{a} p<0.005,{ }^{b} p<0.05$.

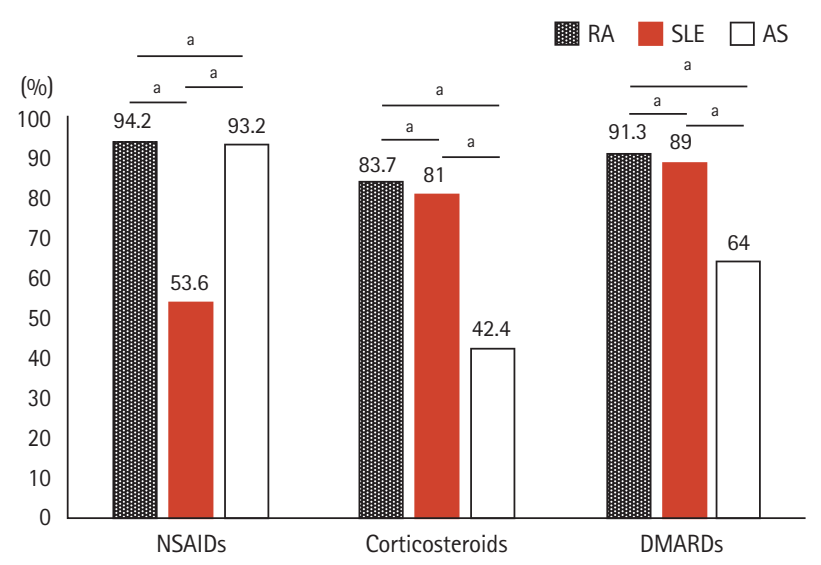

Figure 2. Comparison of rheumatic disease (RD)-related medication use among women of childbearing age (20 to 44 years) with different RDs. NSAID, non-steroidal anti-inflammatory drug; DMARD, disease-modifying anti-rheumatic drug; RA, rheumatoid arthritis; SLE, systemic lupus erythematosus; AS, ankylosing spondylitis. ${ }^{a} p<0.001$. 


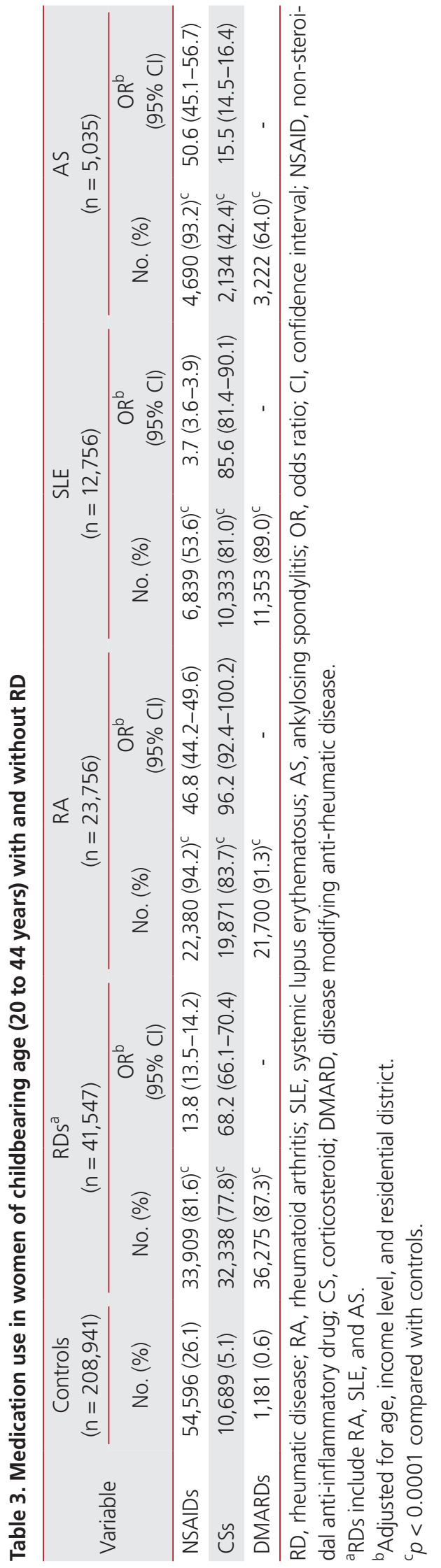

in $87.3 \%$. Significantly more women in the RD group than in the control group were prescribed NSAIDs, CSs, and DMARDs $(p<0.0001$ for all). The RD group had higher odds of taking NSAIDs (OR, 13.8; $95 \% \mathrm{Cl}, 13.5$ to 14.2$)$ and CS (OR, 68.2; $95 \% \mathrm{Cl}, 66.1$ to 70.4 ) than the controls.

Different patterns of RD-related medication use were observed in women with RA, SLE, and AS. Use of NSAIDs was most prevalent in RA (94.2\%), followed by AS (93.2\%) and SLE (53.6\%). Use of CSs was most prevalent in RA (83.6\%), followed by SLE (81.0\%) and AS (42.4\%). In terms of DMARD use, $91.3 \%$ in RA, $89.0 \%$ in SLE, and $64.0 \%$ in AS group were taking DMARDs (Fig. 2). In RA and AS, ORs for using NSAIDs were $46.8(95 \% \mathrm{Cl}, 44.2$ to 49.6$)$ and 50.6 (95\% Cl, 45.1 to 56.7 ), respectively. In SLE, OR for using NSAID was 3.7 (95\% Cl, 3.6 to 3.9), whereas OR for using CSs was $85.6(95 \% \mathrm{Cl}, 81.4$ to 90.1$)$. ORs for using CSs in RA and AS were $96.2(95 \% \mathrm{Cl}, 92.4$ to 100.2$)$ and 15.5 (95\% Cl, 14.5 to 16.4$)$, respectively (Table 3 ).

\section{DISCUSSION}

This study demonstrates that Korean women of childbearing age with RDs had a higher prevalence of comorbidities, including HTN, HLD, DM, and cancer, and had a higher burden of medication use than the general population of the same age and sex.

Women of childbearing age with RDs were three times more likely to have at least one comorbidity, such as HTN, HLD, DM, or cancer, and had a higher risk of developing the comorbidity than age-matched control subjects in this study. This result is expected because patients with RDs are known to have increased risks of CVD and cancer compared with the general population [9,20-22]. The risk of CVD was increased by $48 \%$ in patients with RA compared with the general population [20], and the risk of myocardial infarction was increased with an OR of $1.6(95 \% \mathrm{Cl}, 1.32$ to 1.96$)$ in patients with AS [9]. The CVD risk for patients with SLE has been reported to be 2 to 50 times greater than the risk for the general population $[21,22]$. This increase in CVD risk among patients with RDs results from the combined effects of both non-traditional risk factors (e.g., inflammation, autoantibodies, functional disability) and traditional risk factors (HTN, HLD, DM, obesity, and smoking). The prevalence of HTN, HLD, and type II DM in RA, AS, and SLE was also reported to be higher than in the general population [23- 
25]. Increased overall cancer risk has been demonstrated in patients with SLE (standardized incidence ratio [SIR], 1.28) [26], and AS (hazard ratio [HR], 1.38) [27] compared with those without the disease. In addition, a 10\% increase in overall malignancy risk was shown in patients with RA compared with control subjects [28]. Our findings of increased prevalence of HTN, HLD, DM, and cancer in women of childbearing age with RDs compared with control subjects are comparable to those of previous studies exploring CVD and cancer risk in patients of all ages with RDs.

A notable finding of our study is that relatively young women in their childbearing years had an increased likelihood of having a comorbidity such as HTN, HLD, DM, or cancer. This is in contrast to findings in the general population that the risks of CVD and cancer are higher in men than in women and increase with age $[29,30]$. Although no previous study has evaluated the prevalence of comorbidities in the specific population of women of childbearing age, the risk ages for CVD and cancer were reported to be younger in patients with RDs than in the general population. In RA, the relative risk (RR) for CVD was highest in patients younger than 50 years (RR, 2.59), whereas patients older than 65 years showed the lowest RR of 1.27 compared with the general population [31]. Patients with SLE younger than 40 years had the highest RR for CVD in a retrospective cohort study performed in the UK [32]. In patients with AS, the risk of ischemic heart disease was higher in the newly diagnosed younger age group [33]. Patients younger than 45 years showed greatest SIR (4.11) for cancer in a study of patients with SLE [34], and a similar trend was reported in patients with AS [35].

We cannot explain the increased prevalence of several comorbidities among women with RDs during their childbearing years without evaluating the effect of medication. Several medications used to treat RDs have been shown to promote the development of CVD and cancer. Continuous use of NSAIDs has been demonstrated to increase the incidence of HTN and risk of CVD [36,37]. Use of CSs also increases CVD risk due to their detrimental effects on lipids, glucose tolerance, and HTN [38,39], and higher cumulative doses of CSs are related to the development of lymphoma in patients with SLE (HR, 1.94; 95\% Cl, 1.11 to 3.39) [40]. Some DMARDs such as cyclosporin and azathioprine, have also been associated with an increased risk of malignancy [41]. In addition, medications used to treat RDs can result in reduced fertility [42]. Although the risk is low, NSAIDs can impair fertility [43], and immunosuppressive drugs such as cyclophosphamide can result in permanent ovarian failure [7]. In our study, a high proportion of women in their childbearing years with RDs were taking medications to treat their disease; NSAIDs were prescribed in $81.6 \%$ of patients, CSs in $77.8 \%$, and DMARDs in $87.3 \%$. This high prevalence of medication use in women with RDs in their childbearing years may contribute to the increased prevalence of comorbidities and also significantly affect childbearing by reducing reproductive potential. We found differences in the prevalence of comorbidities and medication use among the different RDs assessed. The prevalence of comorbidities was the highest in patients with SLE, followed by those with RA and AS. This may be related to increased odds for using CSs and DMARDs in patients with SLE and RA compared with those for patients with AS.

This study has several limitations. First, we only analyzed the prevalence of overall cancer, not specific types of prevalent cancer, as this study aimed to assess the burden of comorbidities on this particular population group from a broad perspective. Analysis of the prevalence of specific types of cancer in women of childbearing age with RDs might be the subject of another study. Second, the age- and sex- matched control subjects included patients taking DMARDs for other diseases, such as inflammatory bowel disease, seronegative RA, and non-radiographic spondyloarthropathy, although this proportion accounted for $\leq 0.6 \%$ of the control subjects. Despite these limitations, one strength is that this is the first study to use a nationwide database to estimate the burden of comorbidities and medication use in women of childbearing age with RDs.

In conclusion, Korean women with RDs have a heavy burden of comorbidities and medication use during their childbearing years compared with women without RDs of the same age. Therefore, special attention should be paid to this particular group of women with RDs in their childbearing years when allocating health resources and establishing policies related to comorbidities and medication use. Further research assessing the prevalence of other comorbidities associated with RDs including infection and osteoporosis may provide a more comprehensive understanding of the burden of comorbidities among women of childbearing age with RDs. 


\section{KEY MESSAGE}

1. Women of childbearing age with rheumatic diseases (RDs) showed significantly higher prevalence of comorbidities, including hypertension, hyperlipidemia, diabetes mellitus, and cancer, than the general population of the same age and sex.

2. Use of medications, including nonsteroidal anti-inflammatory drugs, corticosteroids, and disease-modifying anti-rheumatic drugs, was more prevalent in women of childbearing age with RDs than in those without RDs.

3. When allocating health resources and establishing policies, special attention should be paid to this particular group of women of childbearing age with RDs, who carry a heavy burden of comorbidities and medication use.

\section{Conflict of interest}

No potential conflict of interest relevant to this article was reported.

\section{Acknowledgments}

This work was supported by the National Health Insurance Ilsan Hospital grant (2018-20-002). This study used National Health Inform ation Database (NHIS-2020-1-405), created by the National Health Insurance Service. The authors alone are responsible for the content and writing of the paper.

\section{REFERENCES}

1. Krause ML, Makol A. Management of rheumatoid arthritis during pregnancy: challenges and solutions. Open Access Rheumatol 2016;8:23-36.

2. Pons-Estel GJ, Alarcon GS, Scofield L, Reinlib L, Cooper GS. Understanding the epidemiology and progression of systemic lupus erythematosus. Semin Arthritis Rheum 2010;39:257268.

3. Timur $H$, Tokmak A, Turkmen GG, Ali Inal H, Uygur D, Danisman N. Pregnancy outcome in patients with ankylosing spondylitis. J Matern Fetal Neonatal Med 2016;29:2470-2474.

4. Bharti $B$, Lee SJ, Lindsay SP, et al. Disease severity and pregnancy outcomes in women with rheumatoid arthritis: results from the organization of teratology information specialists autoimmune diseases in pregnancy project. J Rheumatol 2015;42:1376-1382.

5. Clowse ME, Magder LS, Witter F, Petri M. The impact of increased lupus activity on obstetric outcomes. Arthritis Rheum 2005;52:514-521.

6. Smith CJ, Bandoli G, Kavanaugh A, Chambers CD. Birth outcomes and disease activity during pregnancy in a prospective cohort of women with psoriatic arthritis and ankylosing spondylitis. Arthritis Care Res (Hoboken) 2020;72:1029-1037.

7. Akawatcharangura P, Taechakraichana N, Osiri M. Prevalence of premature ovarian failure in systemic lupus erythematosus patients treated with immunosuppressive agents in Thailand. Lupus 2016;25:436-444.

8. Gotestam Skorpen C, Hoeltzenbein M, Tincani A, et al. The EULAR points to consider for use of antirheumatic drugs before pregnancy, and during pregnancy and lactation. Ann Rheum Dis 2016;75:795-810.

9. Mathieu S, Pereira B, Soubrier M. Cardiovascular events in ankylosing spondylitis: an updated meta-analysis. Semin Arthritis Rheum 2015;44:551-555.

10. Lindhardsen J, Ahlehoff $\mathrm{O}$, Gislason GH, et al. The risk of myocardial infarction in rheumatoid arthritis and diabetes mellitus: a Danish nationwide cohort study. Ann Rheum Dis 2011;70:929-934.

11. Avina-Zubieta JA, To F, Vostretsova K, De Vera M, Sayre EC, Esdaile JM. Risk of myocardial infarction and stroke in newly diagnosed systemic lupus erythematosus: a general population-based study. Arthritis Care Res (Hoboken) 2017;69:849856.

12. Roos-Hesselink J, Baris L, Johnson M, et al. Pregnancy outcomes in women with cardiovascular disease: evolving trends over 10 years in the ESC Registry Of Pregnancy And Cardiac disease (ROPAC). Eur Heart J 2019;40:3848-3855.

13. Abusief ME, Missmer SA, Ginsburg ES, Weeks JC, Partridge AH. The effects of paclitaxel, dose density, and trastuzumab on treatment-related amenorrhea in premenopausal women with breast cancer. Cancer 2010;116:791-798.

14. Chung MK, Park JS, Lim H, Lee CH, Lee J. Incidence and prevalence of systemic lupus erythematosus among Korean women in childbearing years: a nationwide population-based study. Lupus 2021;30:674-679.

15. Hunter TM, Boytsov NN, Zhang X, Schroeder K, Michaud K, Araujo AB. Prevalence of rheumatoid arthritis in the United States adult population in healthcare claims databases, 20042014. Rheumatol Int 2017;37:1551-1557. 
16. Haroon NN, Paterson JM, Li P, Haroon N. Increasing proportion of female patients with ankylosing spondylitis: a population-based study of trends in the incidence and prevalence of AS. BMJ Open 2014;4:e006634.

17. Song SO, Jung $C H$, Song $Y D$, et al. Background and data configuration process of a nationwide population-based study using the Korean National Health Insurance system. Diabetes Metab J 2014;38:395-403.

18. Cheol Seong S, Kim YY, Khang YH, et al. Data resource profile: the National Health Information database of the National Health Insurance Service in South Korea. Int J Epidemiol 2017:46:799-800.

19. Hwang YJ, Kim N, Yun $C Y$, et al. Validation of administrative big database for colorectal cancer searched by International Classification of Disease 10th Codes in Korean: a retrospective big-cohort study. J Cancer Prev 2018;23:183-190.

20. Avina-Zubieta JA, Thomas J, Sadatsafavi M, Lehman AJ, Lacaille $D$. Risk of incident cardiovascular events in patients with rheumatoid arthritis: a meta-analysis of observational studies. Ann Rheum Dis 2012;71:1524-1529.

21. Hak AE, Karlson EW, Feskanich D, Stampfer MJ, Costenbader $\mathrm{KH}$. Systemic lupus erythematosus and the risk of cardiovascular disease: results from the nurses' health study. Arthritis Rheum 2009;61:1396-1402.

22. Manzi S, Meilahn EN, Rairie JE, et al. Age-specific incidence rates of myocardial infarction and angina in women with systemic lupus erythematosus: comparison with the Framingham Study. Am J Epidemiol 1997;145:408-415.

23. Sabio JM, Vargas-Hitos JA, Navarrete-Navarrete $N$, et al. Prevalence of and factors associated with hypertension in young and old women with systemic lupus erythematosus. J Rheumatol 2011;38:1026-1032.

24. Han C, Robinson DW Jr, Hackett MV, Paramore LC, Fraeman $\mathrm{KH}$, Bala MV. Cardiovascular disease and risk factors in patients with rheumatoid arthritis, psoriatic arthritis, and ankylosing spondylitis. J Rheumatol 2006;33:2167-2172.

25. Urowitz MB, Gladman D, Ibanez D, et al. Clinical manifestations and coronary artery disease risk factors at diagnosis of systemic lupus erythematosus: data from an international inception cohort. Lupus 2007;16:731-735.

26. Song L, Wang Y, Zhang J, Song N, Xu X, Lu Y. The risks of cancer development in systemic lupus erythematosus (SLE) patients: a systematic review and meta-analysis. Arthritis Res Ther 2018;20:270.

27. Sun LM, Muo CH, Liang JA, Chang SN, Sung FC, Kao CH. Increased risk of cancer for patients with ankylosing spondylitis: a nationwide population-based retrospective cohort study. Scand J Rheumatol 2014;43:301-306.

28. Simon TA, Thompson A, Gandhi KK, Hochberg MC, Suissa $S$. Incidence of malignancy in adult patients with rheumatoid arthritis: a meta-analysis. Arthritis Res Ther 2015;17:212.

29. Merz AA, Cheng $S$. Sex differences in cardiovascular ageing. Heart 2016;102:825-831.

30. White MC, Holman DM, Boehm JE, Peipins LA, Grossman M, Henley SJ. Age and cancer risk: a potentially modifiable relationship. Am J Prev Med 2014;46(3 Suppl 1):S7-S15.

31. Fransen J, Kazemi-Bajestani SM, Bredie SJ, Popa CD. Rheumatoid arthritis disadvantages younger patients for cardiovascular diseases: a meta-analysis. PLoS One 2016;11:e0157360.

32. Rees F, Doherty M, Grainge M, Lanyon P, Davenport G, Zhang W. Burden of comorbidity in systemic lupus erythematosus in the UK, 1999-2012. Arthritis Care Res (Hoboken) 2016;68:819-827.

33. Huang YP, Wang YH, Pan SL. Increased risk of ischemic heart disease in young patients with newly diagnosed ankylosing spondylitis: a population-based longitudinal follow-up study. PLoS One 2013;8:e64155.

34. Tallbacka KR, Pettersson T, Pukkala E. Increased incidence of cancer in systemic lupus erythematosus: a Finnish cohort study with more than 25 years of follow-up. Scand J Rheumatol 2018;47:461-464.

35. Chang CC, Chang CW, Nguyen PA, et al. Ankylosing spondylitis and the risk of cancer. Oncol Lett 2017;14:1315-1322.

36. Coxib and traditional NSAID Trialists' (CNT) Collaboration, Bhala N, Emberson J, et al. Vascular and upper gastrointestinal effects of non-steroidal anti-inflammatory drugs: meta-analyses of individual participant data from randomised trials. Lancet 2013;382:769-779.

37. Morrison A, Ramey DR, van Adelsberg J, Watson DJ. Systematic review of trials of the effect of continued use of oral non-selective NSAIDs on blood pressure and hypertension. Curr Med Res Opin 2007;23:2395-2404.

38. laccarino L, Bettio S, Zen M, et al. Premature coronary heart disease in SLE: can we prevent progression? Lupus 2013;22:1232-1242.

39. Panoulas VF, Douglas KM, Stavropoulos-Kalinoglou A, et al. Long-term exposure to medium-dose glucocorticoid therapy associates with hypertension in patients with rheumatoid arthritis. Rheumatology (Oxford) 2008;47:72-75.

40. Bernatsky S, Ramsey-Goldman R, Joseph L, et al. Lymphoma risk in systemic lupus: effects of disease activity versus treatment. Ann Rheum Dis 2014;73:138-142. 
41. De Cock D, Hyrich K. Malignancy and rheumatoid arthritis: epidemiology, risk factors and management. Best Pract Res Clin Rheumatol 2018;32:869-886.

42. Ostensen M. Sexual and reproductive health in rheumatic disease. Nat Rev Rheumatol 2017;13:485-493.
43. Micu MC, Micu R, Ostensen M. Luteinized unruptured follicle syndrome increased by inactive disease and selective cyclooxygenase 2 inhibitors in women with inflammatory arthropathies. Arthritis Care Res (Hoboken) 2011;63:1334-1338. 